\title{
Liposomes and nanotechnology in drug development: focus on oncotargets
}

\author{
This article was published in the following Dove Press journal: \\ International Journal of Nanomedicine \\ 13 September 2012 \\ Number of times this article has been viewed
}

\section{Tomohiro Kozako' \\ Naomichi Arima ${ }^{2}$ \\ Makoto Yoshimitsu ${ }^{3}$ \\ Shin-Ichro Honda' \\ Shinji Soeda'}

'Department of Biochemistry, Faculty of Pharmaceutical Sciences, Fukuoka University, Fukuoka, Japan; ${ }^{2}$ Division of Hematology and Immunology, Center for Chronic Viral Diseases, Graduate School of Medical and Dental Sciences, Kagoshima University, Kagoshima, Japan; ${ }^{3}$ Department of Hematology and Immunology, Kagoshima University Hospital, Kagoshima, Japan
Correspondence: Tomohiro Kozako Department of Biochemistry, Faculty of Pharmaceutical Sciences, Fukuoka University, 8-19-I Nanakuma, Jonan-ku, Fukuoka 8I4-0I80, Japan $\mathrm{Tel}+8|9287| 663$ |

Fax+8I 92862443

Email kozako@fukuoka-u.ac.jp
Abstract: Nanotechnology is the development of an engineered device at the atomic, molecular, and macromolecular level in the nanometer range. Advances in nanotechnology have proven beneficial in therapeutic fields such as drug-delivery and gene/protein delivery. Antigen delivery systems are important for inducing and modifying immune responses. In cellular immunity, cytotoxic T lymphocytes (CTLs) are important in the host defense against tumors. Key to the development of CTL-inducible vaccines is the ability to deliver antigens to antigen-presenting cells efficiently and to induce the subsequent activation of $\mathrm{T}$ cell-mediated immunity without adjuvants, as they can induce excessive inflammation leading to systemic febrile disease. Since expression and cloning methods for tumor-associated antigens have been reported, cancer vaccines that induce effective cell immunity may be promising therapeutic candidates, but Th2 cells are undesirable for use in cancer immunotherapy. Peptide vaccines have immunological and economic advantages as cancer vaccines because CTL epitope peptides from tumorassociated antigens have high antigen-specificity. However, cancer vaccines have had limited effectiveness in clinical responses due to the ability of cancer cells to "escape" from cancer immunity and a low efficiency of antigen-specific CTL induction due to immunogenic-free synthetic peptides. In contrast, carbohydrate-decorated particles such as carbohydrate-coated liposomes with encapsulated antigens might be more suitable as antigen delivery vehicles to antigen-presenting cells. Oligomannose-coated liposomes (OML) can eliminate established tumors in mouse cancer models. In addition, OMLs with an encased antigen can induce antigenspecific CTLs from peripheral blood mononuclear cells obtained from patients. Feasibility studies of OML-based vaccines have revealed their potential for clinical use as vaccines for diseases where CTLs act as effector cells. Furthermore, use of the hepatitis B core particle, in which tumor-antigen epitopes are set, has consistently been shown to induce strong CTL responses without the use of an adjuvant. Thus, nanoparticles may provide a new prophylactic strategy for infectious disease and therapeutic approaches for cancer via the induction of T-cell immunity.

Keywords: adult T cell leukemia, cytotoxic T lymphocytes, oligomannose-coated liposomes, vaccine

\section{Introduction}

Recent developments in multifunctional nanoparticles offer a great potential for targeted delivery of therapeutic compounds and imaging contrast agents to specific cell types, enhancing therapeutic effects and minimizing side effects. For pharmaceutical purposes, nanoparticles are defined as solid colloidal particles ranging in size from $10 \mathrm{~nm}$ to $400 \mathrm{~nm}$. They consist of macromolecular materials in which the active agent (drug or biologically active material) is dissolved, entrapped, and/or encapsulated, or 
to which the active agent is adsorbed or attached. ${ }^{1}$ The ability to target liposomal drugs to tumors or target cells represents an attractive strategy for developing more effective cancer therapies.

Antigen-specific $\mathrm{CD}^{+}$cytotoxic $\mathrm{T}$ lymphocyte (CTL) induction is an attractive immunotherapeutic strategy against hematological malignancies, other cancers, and infectious diseases. ${ }^{2-4}$ Cluster of differentiation (CD) molecules are expressed on most normal cells. However, CD molecules are often overexpressed on tumor cells. Viral-specific and mutated antigens are clearly foreign, and viral antigens are present only on tumors that are virally induced. Therefore, CTL responses may be initiated against overexpressed cell surface markers or viral antigens expressed by major histocompatibility complex (MHC) molecules to target tumor cells. For instance, impaired host CTL function abrogates protection against the accumulation of human T-cell leukemia virus-1 (HTLV-1)-transformed cells. Thus, preventing the loss of, or inducing host CTL functions, may yield an effective immune strategy against leukemogenesis such as adult T-cell leukemia-lymphoma (ATL). ${ }^{5,6}$ In particular, $\mathrm{CD}^{+} \mathrm{T}$ lymphocytes differentiate into distinct $\mathrm{T}$ helper (Th) cell subsets depending on the type of disease. Among these effector Th subsets, Th1 cells support the expansion and persistence of CTLs, but Th2 cells are undesirable for immunotherapy of cancer or for vaccination against intracellular pathogens. Therefore, a potent vaccine for these diseases should activate both $\mathrm{CD}^{+}$and $\mathrm{CD}^{+}{ }^{+} \mathrm{T}$ lymphocytes to generate antigen-specific CTLs and Th1 cells, respectively.

The difficulty of inducing antigen-specific CTLs in individual patients vitiates the more widespread use of adoptive $\mathrm{T}$ cell therapy. Liposomes can encapsulate large and broad antigenic sequences, immunomodulatory factors, and drugs, and thus can serve as potent delivery vehicles. Whereas free synthetic peptides have proven to be relatively poor immunogens, the use of peptides with oligomannosecoated liposomes (OML) can induce strong cellular immunity. ${ }^{7-10}$ Virus-like particles (VLPs) have also been shown to induce strong CTLs, even without adjuvant. ${ }^{11,12}$ Furthermore, liposome-protamine-DNA (LPD) nanoparticles are currently being employed as effective peptide carriers. ${ }^{13}$ The immunostimulatory and adjuvant properties of LPD nanoparticles have made them suitable for delivering antigens for the purpose of vaccination. ${ }^{14}$

Thus, the use of liposomes and VLPs to target drugs to tumors represents an attractive therapeutic strategy, especially when used with convenient targeting moieties such as peptides. This review presents an overview of the current attempts to use nanoparticles for vaccines.

\section{Liposomal vaccines}

In the study of potent immune responses, adjuvants are crucial. However, adjuvants can induce unfavorable immune responses such as systemic fever, hepatotoxicity, and hypersensitivity "flare reactions" around the injection site. Liposome co-encapsulated $\mathrm{CpG}$ oligodeoxynucleotides with recombinant Leishmania major stress-inducible protein 1, enhanced immune responses, and protection against leishmaniasis in immunized BALB/c mice when compared with $\mathrm{CpG}$ given in soluble form with antigen or antigen without adjuvant. ${ }^{15}$ This study indicated the superiority of $\mathrm{CpG}$ oligodeoxynucleotides in its liposomal form compared to the soluble form, and that it has an important role in vaccine development strategies against leishmaniasis. ${ }^{16}$

LPD nanoparticles are spheres (around $150 \mathrm{~nm}$ ) generated by spontaneous rearrangement of a lipid shell composed of 1,2-dioleoyl-3-trimethylammonium-proprane and cholesterol around a protamine-condensed DNA to develop a virus-like structure. ${ }^{17}$ The immunostimulatory and adjuvant properties of LPD nanoparticles have made them suitable for delivering antigens for the purpose of vaccination. ${ }^{14}$ The small diameter and positively charged characteristics of LPD nanoparticles allow lymphatic delivery of peptides and dendritic cell (DC) targeting. ${ }^{18,19}$

\section{Implications of antigen delivery to antigen-presenting cells (APCs) by carbohydrate-coated liposomes}

The critical requirements for an antigen delivery system for vaccines include efficient delivery to the cell of choice, the ability to load the antigen onto MHC molecules, and the ability to activate APCs to express costimulatory molecules to aid the induction of CTL responses. Liposomes have been widely exploited as antigen delivery systems for treating a variety of diseases. These vesicles can be prepared in various ways, which may affect the immunogenicity of the encapsulated antigens. ${ }^{20}$ Efficient delivery of antigens to immune cells can be facilitated by agents that bind selectively to molecular structures on the surface of the targeted cells. The conjugation of liposomes with antibodies directed to cell surface receptors, recombinant ligand proteins, or chemical ligands, such as carbohydrates, can facilitate uptake by specific cells and therefore have been tested for targeting liposomes to cells and tissues. ${ }^{21-23}$ The recognition and phagocytosis of pathogens and their subsequent destruction 
is an important mechanism of immune defense. APCs express many C-type lectin receptors (CLRs) that act as phagocytic receptors. ${ }^{24,25}$ Some CLRs contain internalization motifs in their cytoplasmic domains, which direct the uptake of ligands and subsequent sorting of CLRs and attached cargo into late endosomes. CLRs also contain an immunoreceptor tyrosine-based activation motifs or immunoreceptor tyrosinebased inhibitory motifs in their cytoplasmic domains that trigger or modulate distinct signaling pathways to induce the expression of specific cytokines, which then determine T cell polarization. ${ }^{25}$ CLRs recognize distinct carbohydrate structures expressed on pathogens, and therefore the use of carbohydrates that are preferentially recognized by CLRs as targeting signals may be a more sophisticated alternative. ${ }^{26}$ Thus, carbohydrate-decorated particles such as carbohydratecoated liposomes with encapsulated antigens may be more suitable antigen delivery vehicles to target CLRs on APCs.

Molecular structures containing terminal mannose are uncommon elements of mammalian tissues, but abundant and highly conserved in microorganisms. Mannose-recognizing CLRs can facilitate the binding and endocytosis of ligands with terminal mannose, fucose, and $\mathrm{N}$-acetylglucosamine. ${ }^{27}$ Mannose residues can be recognized by DC-specific intracellular adhesion molecule-3-grabbing nonintegrin (DC-SIGN, CD209) family molecules expressed by APCs, langerin (CD207) on Langerhans cells and DCs and by dectin- 2 on monocytes or macrophages. ${ }^{28,29}$ Thus, carbohydrate-modified antigens may be preferentially delivered to APCs due to DC-SIGN-mediated uptake of antigens, resulting in effective antigen presentation to T cells. ${ }^{30}$ Therefore, mannose residues attached to antigens or as a coating on antigen-carrying liposomes have promise for the induction of immune responses using the carbohydrate recognition pathways of APCs.

\section{Oligomannose-coated liposomes as a drug delivery system}

Kojima et al have developed a new active agent (drug, peptide, or biologically active material) delivery system for the control of diseases such as infection, cancer and allergy, using OMLs. ${ }^{31-34}$ OMLs were prepared from dipalmitoylphosphatidylethanolcholine, cholesterol, and mannotriose-dipalmitoylphosphatidylethanolamine at a molar ratio of 10:10:1 and extruded through a $1-\mu \mathrm{m}$ pore membrane (Figure 1). This approach relies on the preferential recognition of oligomannose residues on OMLs by APCs expressing APC-specific mannose-binding lectin receptors, and subsequent uptake of OMLs and antigens encased in the

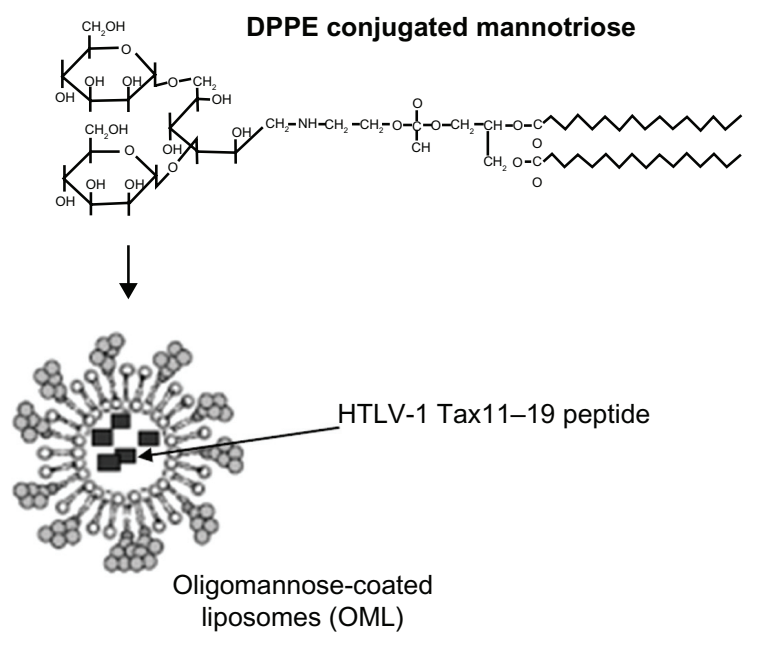

Figure I Structure of synthetic neoglycolipids consisting of dipalmitoylphosphatidylethanolamine.

Abbreviation: DPPE, dipalmitoylphosphatidylethanolcholine; HTLV-I, human T-cell leukemia virus-I.

OMLs to be loaded on MHC class I and class II molecules. ${ }^{7}$ OMLs can also activate and induce the maturation of APCs with enhanced expression of costimulatory molecules and proinflammatory cytokines. $^{9}$

In the efficient induction of CTLs, Th1 cells support the expansion and persistence of CTLs, whereas Th2 cells are undesirable for immunotherapy of cancer or vaccination against intracellular pathogens. OMLs can enhance interleukin (IL)-12 production and the expression of costimulatory molecules (CD40, CD80, CD86, and MHC class II molecules) on peritoneal macrophages. ${ }^{9}$ Interestingly, the production of inflammatory cytokines such as IL-1 and IL-6 from either DCs or macrophages was suppressed in response to OML incorporation. ${ }^{9}$ However, spleen cells from mice immunized with ovalbumin (OVA)-containing OMLs (OML/OVA) produced significant levels of interferon (IFN) $-\gamma$ that exceeded IL-5 production when stimulated with OVA in vitro. In contrast, spleen cells predominantly produced IL-5 over IFN- $\gamma$ in response to OVA stimulation, if mice were immunized with OVA adsorbed on aluminum hydroxide, an adjuvant for the induction of Th2 immune responses. Thus, to induce antigen-specific Th1 cells, a suitable adjuvant must be used in conjunction with OVA incorporation.

Pathogens share similar structures termed pathogenassociated molecular patterns (PAMPs). Pattern recognition receptors (PRRs) such as Toll-like receptors (TLRs) are a primitive part of the immune system. ${ }^{35}$ Binding of PAMPs to TLRs triggers signal transduction events that activate mitogen-activated protein kinases and transcription factors such as nuclear factor- $\kappa \mathrm{B}$, leading to the induction of 
inflammatory cytokines such as IL-1 $\beta$, IL-6, IL-12, and tumor necrosis factor- $\alpha .{ }^{36}$ OML-stimulated APCs preferentially produce IL-12, but suppress the production of IL-6 and IL-1 $\beta$. SIGNR1 colocalizes in lipid rafts with several Src family kinases, such as Lyn, which modulate the maturation of DCs. ${ }^{37}$ Therefore, OMLs may activate APCs through particular signaling pathways distinct from those triggered by interactions between PAMPs and TLRs.

\section{Oligomannose-coated liposomes as a therapeutic vaccine}

Kojima et al demonstrated that OMLs can be used as therapeutic vaccines that induce CTLs specific to the encased antigen without adjuvant (Table 1). ${ }^{38}$ Immunization of BALB/c mice with OMLs encasing soluble leishmanial antigen protected against subsequent Leishmania major infection due to the predominant induction of leishmanial antigen-specific Th1 immune responses over Th2 immune responses. Preliminary studies have also examined the effect of OML-based vaccines using soluble protozoan lysates of Toxoplasma gondii, Trypanosoma brucei gambiense, Babesia rodhaini, or Neospora caninum on the corresponding protozoal infections in mice. ${ }^{33}$ OML-based vaccinations, incorporating a single injection of OMLs containing $1 \mu \mathrm{g}$ of antigen, induced antitumor activity in an E.G7-OVA tumor-bearing mouse model that suppressed tumor growth and led to tumor rejection, where approximately $50 \%$ of the mice showed elimination of an established tumor. ${ }^{32}$ Recently, Kojima et al reported the induction of CTLs specific for either HLA-A24-restricted epitopes of survivin2B using mixed lymphocyte-peptide cultures with OML-coated survivin2B peptides or human papillomavirus type16 E6 and E7 with OML-coated papillomavirus DNA. ${ }^{38,39}$ Therefore, OMLs are suitable for use as safe vaccine carriers of peptides and plasmid DNA and as adjuvants.

OML with an encased allergen may also have an antiallergic effect since IFN- $\gamma$ produced by Th1 cells induced by OML-based vaccination inhibited the development and activation of allergen-specific Th2 cells, which promotes IgE synthesis and mediates type I allergic reactions. Immunization of OMLs with entrapped Cry j 1, identified as major allergen in Japanese cedar pollen, prevents elevation of serum IgE levels elicited by Cry $\mathrm{j} 1$ administration in both unsensitized mice and Cry j 1-presensitized mice. This indicates that OML-encased allergens may serve as immunotherapeutic agents to control type I allergic diseases. These inhibitory effects may occur due to a shift from Th2 immune responses to allergen-specific Th1 immune responses since Cry j 1-specific IgG1 production mediated by Th2 cells was significantly reduced, but Cry j 1 -specific IgG2a production mediated by Th1 cells increased in the sera of OML-based vaccinated mice. ${ }^{31}$ Nasal administration of OMLs can induce entrapped HA-specific secretory IgA in local tissues and OVA-specific serum IgG and IgA. ${ }^{40}$ These feasibility studies of OML-based vaccines have revealed their potential for clinical use as vaccinations for diseases where CTLs and/or Th1 cells are effectors.

\section{Prophylactic nanoparticle vaccines for adult $\mathbf{T}$ cell leukemia}

ATL is an aggressive malignancy of mature peripheral T-lymphocytes. ${ }^{41-43}$ Despite recent progress in chemotherapy and supportive care for hematological malignancies, the median survival time is still only approximately 13 months. ${ }^{44-46}$ Therefore, new strategies for the therapy and

Table I Therapeutic efficiency of oligomannose-coated liposome-based vaccines

\begin{tabular}{|c|c|c|c|}
\hline Encapsulated antigen & Responses & Sources & Reference \\
\hline \multirow[t]{3}{*}{ OVA } & Rejection of EG7-OVA tumor (pre-immunization) & C57BL/6 & 32 \\
\hline & Elimination of established EG7-OVA tumor (post-immunization) & C57BL/6 & 7 \\
\hline & Induction of antigen-specific ThI cells and CTLs & Balb/c & 40 \\
\hline Crude extract of Leishmania major & Suppression of foodpad swelling by Leishmania major infection & $\mathrm{Balb} / \mathrm{c}$ & 35 \\
\hline Recombinant NcGRA7 & Protection of dams and offspring from Neospora caninum infection & $\mathrm{Balb} / \mathrm{c}$ & 34 \\
\hline $\begin{array}{l}\text { Recombinant apical membrane } \\
\text { antigen }\end{array}$ & Reduction of offspring mortality from Neospora caninum infection & $\mathrm{Balb} / \mathrm{c}$ & 34 \\
\hline Cry j I & $\begin{array}{l}\text { Prevention of lgE elevation in sera in response to Cry j I } \\
\text { sensitization }\end{array}$ & $\mathrm{Balb} / \mathrm{c}$ & 31 \\
\hline $\begin{array}{l}\text { HLA-A*2402-restricted survivin2B } \\
\text { peptide }\end{array}$ & Induction of survivin-specific CTLs & PBMCs from patients & 38 \\
\hline HPV I6 E6 and E7 plasmid DNA & Induction of human HPV-specific CTLs & PBMCs from carriers & 39 \\
\hline HLA-A*020I-restricted HTLV-I & Induction of HTLV-I specific CTLs & HLA-A*020I-Tg mice & 10 \\
\hline Tax peptide & Induction of HTLV-I specific CTLs & PBMCs from HTLV-I carriers & 10 \\
\hline
\end{tabular}

Abbreviations: NcGRA7, Neospora caninum dense granule protein 7; PBMCs, peripheral blood mononuclear cells; Cry j I, Japanese cedar pollen; OVA, ovalbumin; CTLs, cytotoxic T lymphocytes; HTLV, human T-cell leukemia virus-I; Th, T helper; HLA, human leukocyte antigen; HPV, Human papilloma virus. 
prophylaxis of ATL (eg, vaccines and novel molecular target agents) are urgently needed. Although the clonal evolution of ATL cells may involve multiple steps,${ }^{47}$ an insufficient $T$ cell response to HTLV-1 is a potential risk factor. ${ }^{48}$ HTLV-1specific CTLs are critical in the host immune response against HTLV-1 infection. ${ }^{49,50}$ We previously reported a decreased frequency and function of HTLV-1 Tax-specific CD ${ }^{+}$ $\mathrm{T}$ cells in ATL patients and the upregulation of the negative immunoregulatory programmed death 1 marker on HTLV-1 Tax-specific CTLs from asymptomatic HTLV-1 carriers and ATL patients. ${ }^{51-54}$ Impaired host CTL function reduces protection against the accumulation of HTLV-1-transformed cells; thus, targeting this mechanism may yield an effective immune strategy against leukemogenesis. HTLV-1 Tax-targeted vaccines in a rat model of HTLV-1-induced lymphomas showed promising antitumor effects. ${ }^{55}$ Therefore, HTLV-1-specific CTLs are important for the immunological suppression of HTLV-1-infected cell proliferation and pathogenesis of ATL.
The difficulty in inducing antigen-specific CTLs in individual patients suggests why the use of adoptive $\mathrm{T}$ cell therapy is not widespread. OMLs can be an effective antigen delivery system for cancer immunotherapy by activating CTLs and Th subsets. ${ }^{56,57}$ We examined the efficient induction of HTLV-1-specific $\mathrm{CD}^{+} \mathrm{T}$ cell responses by OMLs encapsulating the HLA-A*0201-restricted HTLV-1 Tax-epitope (OML/Tax; Figure 1). Immunization of HLA-A*0201 transgenic mice with OML/Tax induced HTLV-1-specific IFN- $\gamma$ production from $\mathrm{T}$ cells, whereas immunization with the epitope peptide alone did not (Figure 2A). DCs exposed to OML/Tax showed increased expression of CD86 and MHC class I, and class II molecules such as HLA-A02. ${ }^{10}$ In addition, HTLV-1-specific CD8 ${ }^{+}$ $\mathrm{T}$ cells were efficiently induced by OML/Tax derived from HTLV-1 carriers compared with the epitope peptide alone (Figure 2B). OML/Tax increased the number of HTLV-1specific $\mathrm{CD}^{+} \mathrm{T}$ cells up to 1400 -fold, while treatment with peptide alone and without antigen showed increases of

A
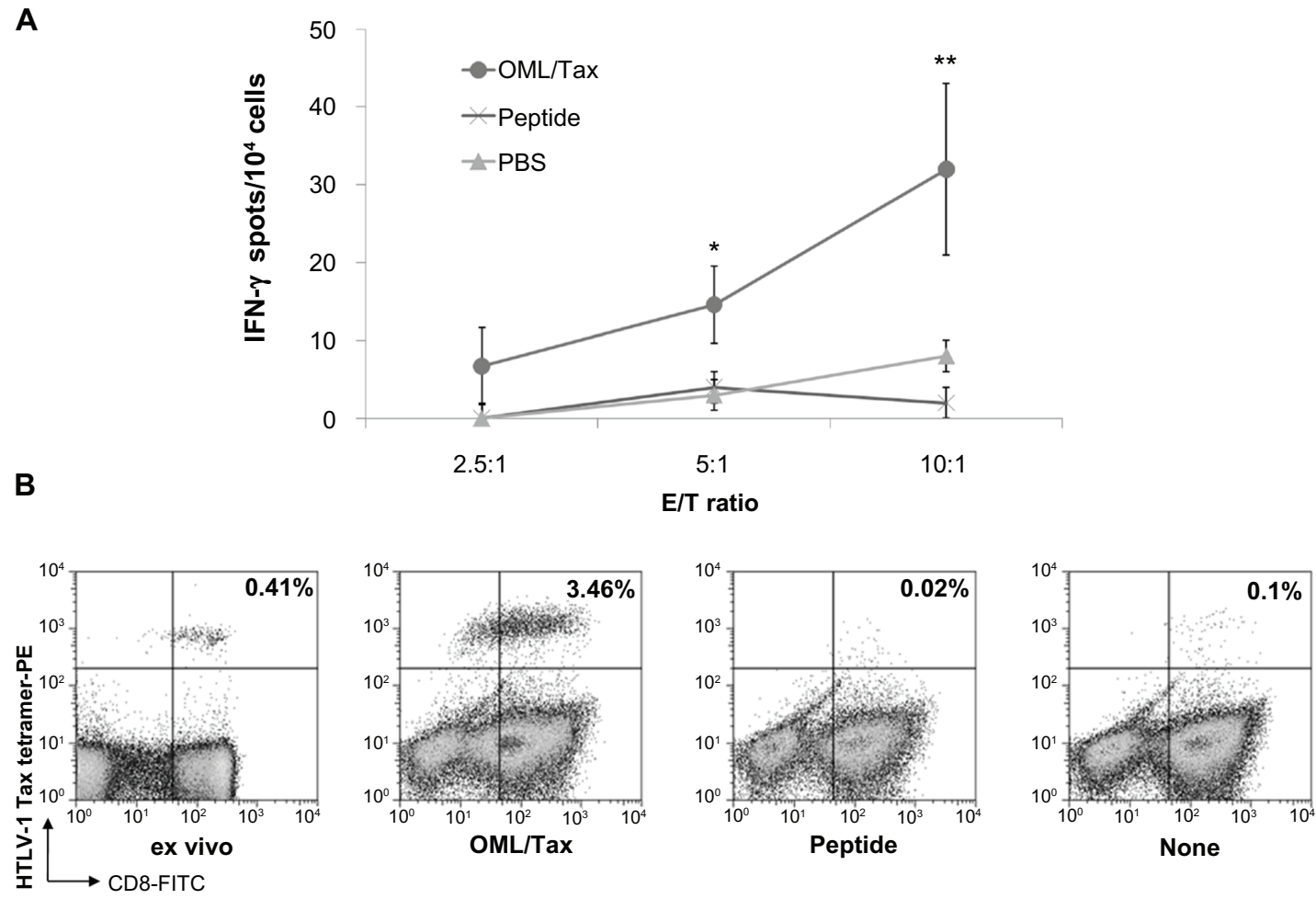

Figure 2 Induction of cellular immunity by intradermal immunization of mice with OML/Tax. (A) HLA-A*020I transgenic mice ( $n=5$ per group) were intradermally immunized twice with OML/Tax, HTLV-I peptide (LLFGYPVYV), or PBS on days 0 and I4. Seven days after the last immunization, the spleens and inguinal lymph nodes were collected. Inguinal lymph node cells $\left(2 \times 10^{6} /\right.$ well) were stimulated in vitro with HTLV-I peptide. Six days later, the number of IFN- $\gamma$-producing cells per 2.5 , 5 , or $10 \times 10^{4}$ inguinal lymph node cells upon stimulation with syngeneic bone marrow derived-DCs $\left(I \times 10^{4} /\right.$ well, pulsed with or without each peptide) were determined using an ELISPOT assay. IFN- $\gamma$ spots were expressed as the number of peptide-loaded to peptide-unloaded target cells. $* P<0.05$; $* * P<0.0$ I vs PBS group. All experiments were performed in triplicate. Values represent the mean from five mice. Results represent the mean \pm SD. (B) Freshly isolated or cryopreserved PBMCs from HTLV-I carriers were cultured with OML/Tax, peptide alone, or without antigen. The tetramer assay was performed using fresh (ex vivo) or cultured PBMCs. The numbers in the upper right quadrants represent the percentage of tetramer ${ }^{+} \mathrm{CD} 8^{+} \mathrm{T}$ cells within the $\mathrm{T}$ lymphocyte population.

Abbreviations: DCs, dendritic cells; FITC, fluorescein isothiocyanate; IFN, interferon; HTLV-I, human T-cell leukemia virus-I; OML, oligomannose-coated liposome; PBS, phosphate-buffered saline; PE, phycoerythrin; PBMCs, peripheral blood mononuclear cells. 
95- and 35-fold, respectively. Furthermore, these HTLV-1specific $\mathrm{CD}^{+}$cells induced apoptosis of HTLV-1 epitope peptide-pulsed T2-A2 cells. $\mathrm{CD}^{+} \mathrm{T}$ cells efficiently lysed Tax peptide-loaded T2-A2 cells, whereas only low-level background lysis was observed in the absence of Tax peptide and for CMV peptide-loaded T2-A2 cells. These results suggest that OML/Tax induces antigen-specific cellular immune responses without the need for adjuvants and may be an effective vaccine carrier for prophylaxis of tumors and infectious diseases. ${ }^{10}$

Whereas free synthetic peptides have proven to be relatively poor immunogens, VLPs, such as hepatitis B core ( $\mathrm{HBc})$, have consistently been shown to induce strong antibody and CTL responses, even without an adjuvant. ${ }^{11,12}$ $\mathrm{HBcAg}$, a potent immunogen that elicits strong humoral, T-helper, and CTL responses and is amenable to a variety of heterologous epitopes without an adjuvant, ${ }^{58}$ may be an effective carrier protein for use in T-cell mediated vaccine development. ${ }^{59}$ We fused the HTLV-1 Tax11-19 peptide, recognized by HLA-A*0201-restricted HTLV-1-specific $\mathrm{CD}^{+} \mathrm{T}$-cells with high frequency, ${ }^{52}$ to $\mathrm{HBcAg}$ to yield an HTLV-1/HBc chimeric particle. A synthetic DNA fragment, the HTLV-1 Tax sequence from amino acid positions 8-22, including the Tax11-19 epitope recognized by HLA-A*0201, was inserted into the HBc gene. The chimeric protein was expressed in Pichia pastris KM71. The chimeric particle maintained the capacity to fold correctly and spontaneously assembled into structured capsid particles with a diameter of $36 \mathrm{~nm}$. Immunization of HLA-A*0201 transgenic mice with the chimeric particle induced antigen-specific IFN- $\gamma$ production as assessed by enzyme-linked immunospot assays, whereas immunization with the epitope peptide alone induced no reaction (Figure $3 \mathrm{~A}$ ). Immunization with the chimeric particle also induced HTLV-1-specific CD8 ${ }^{+}$T-cells

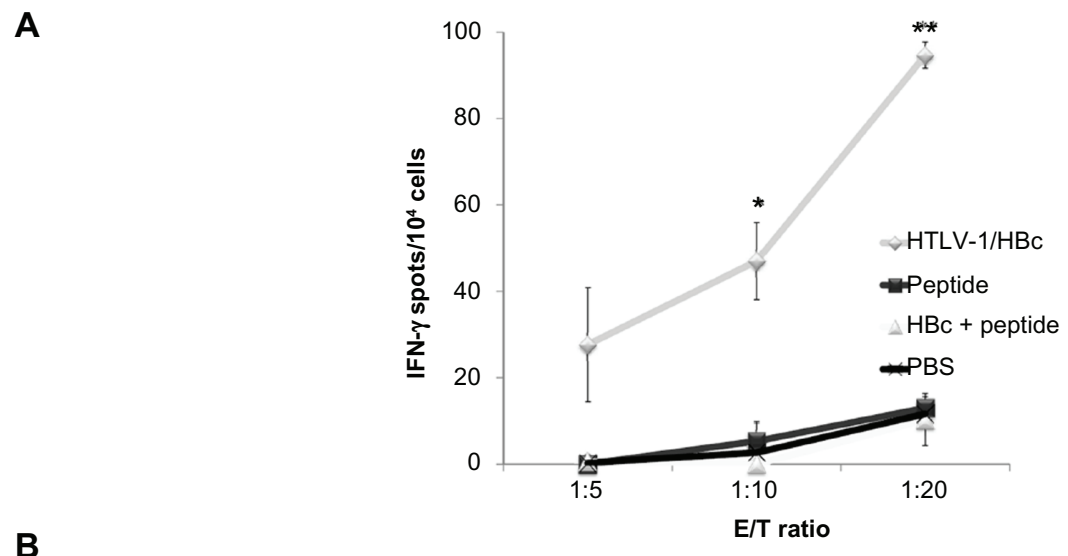

B

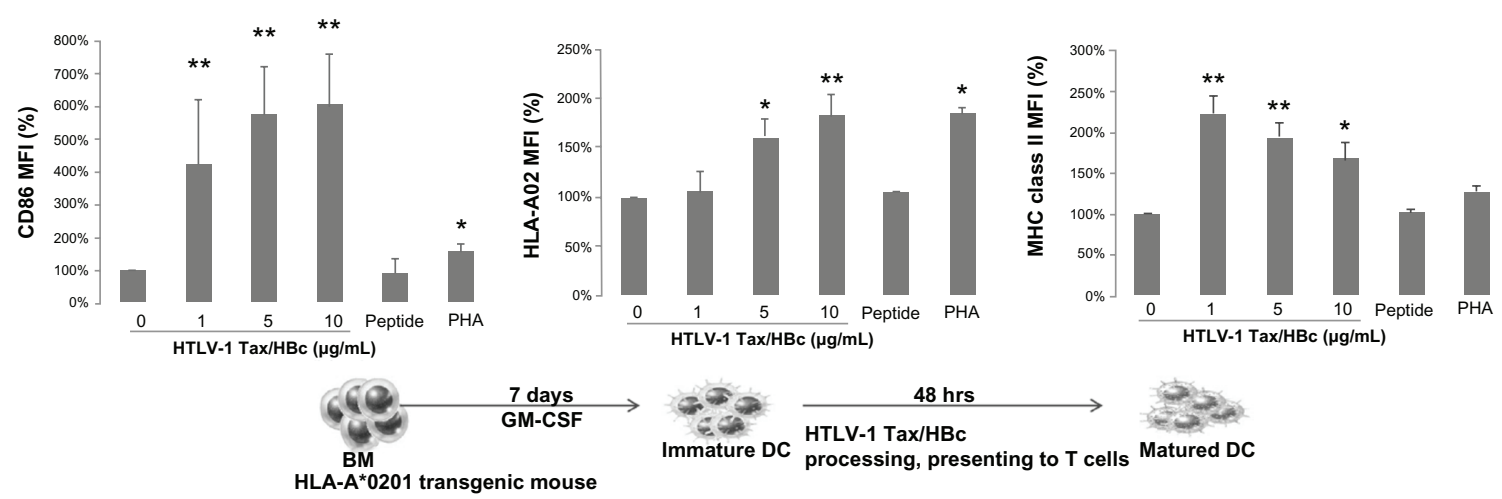

Figure 3 The induction of cellular immunity by intradermal immunization with HTLV-I Tax/HBc chimeric particles. (A) HLA-A*020I transgenic mice were intradermally immunized twice with HTLV-I Tax/HBc chimeric particles $(20 \mu \mathrm{g})$, HTLV-I peptide (I $\mu \mathrm{g}:$ LLFGYPVYV), HTLV-I peptide (I $\mu \mathrm{g})$ plus HBc particles (20 $\mu \mathrm{g})$ or PBS at day 0 and day 14. Seven days after the last immunization, the spleens and inguinal lymph nodes were collected. The inguinal lymph node cells $\left(2 \times 10^{6} /\right.$ well $)$ were stimulated with Tax I -19 peptide in vitro. Six days later, the frequency of cells producing IFN- $\gamma$ per 5,10 , or $20 \times 10^{4}$ inguinal lymph node cells upon stimulation with syngenic bone marrow-derived DCs $\left(I \times 10^{4} /\right.$ well $)$, pulsed with or without each peptide, was determined by ELISPOT assay. IFN- $\gamma$ spots are expressed as the number of peptide-loaded to peptide-unloaded target cells. $* P<0.05 ; * * P<0.01$ vs PBS group. The experiments were performed in triplicate. Results represent the mean \pm SD. (B) Maturation of DCs induced by HTLV-I Tax/HBc chimeric particles was assessed by the expression of CD86 and HLA-02 on the surface of DCs after incubation with antigens. Murine immature dendritic cells (iDCs) were obtained from bone marrow (BM) precursors. iDCs were incubated with the indicated concentrations of $\mathrm{HTLV}$-I Tax/HBc chimeric particles: $10 \mu \mathrm{g} / \mathrm{mL}$ of HTLV-I Tax peptide or $10 \mu \mathrm{g} / \mathrm{mL}$ of phytohemagglutinin (PHA) at $37^{\circ} \mathrm{C}$. Data are expressed as the mean fluorescence intensity (MFI) for each molecule compared to unpulsed $(0 \mu \mathrm{g} / \mathrm{mL})$ iDC controls. Results represent means \pm SD of four independent experiments. $* P<0.05 ; * * P<0.0 \mathrm{I}$ vs unpulsed iDC controls.

Abbreviations: DCs, dendritic cells; IFN, interferon; HBC, hepatitis B core; PBS, phosphate-buffered saline. 
in the spleen and inguinal lymph nodes.$^{60}$ Furthermore, DCs isolated from HLA-A*0201-transgenic mice exposed to the chimeric particle showed increased expression of CD86, HLA-A02, and MHC class II (Figure 3B). In addition, our results demonstrated that $\mathrm{HTLV}-1$-specific $\mathrm{CD} 8^{+} \mathrm{T}$ cells could be induced by a peptide with HTLV-1/HBc particles from ATL patients, but not by the peptide or HTLV-1/HBc particles alone, and could lyse immune cells presenting the peptide. ${ }^{60}$ This suggests that HTLV-1/HBc chimeric particles can induce strong cellular immune responses without the need for an adjuvant via the effective maturation of DCs. Previous studies have demonstrated the efficient processing of lymphocytic choriomeningitis virus-derived p33/HBc chimera via cross-presentation, although only weak CTL responses were induced in C57BL/6 mice. ${ }^{59}$ Thus, while VLPs alone are inefficient at inducing CTL responses, they become potent vaccines when combined with APC-activating substances such as anti-CD40 mAbs or nonmethylated CG motif-rich DNA (CpGs). These results suggest that the HTLV-1/HBc chimeric particle can induce strong cellular immune responses without adjuvants by inducing the maturation of DCs and is potentially useful as an effective carrier for therapeutic vaccines in tumors or in infectious diseases by substituting the epitope peptide..$^{60}$

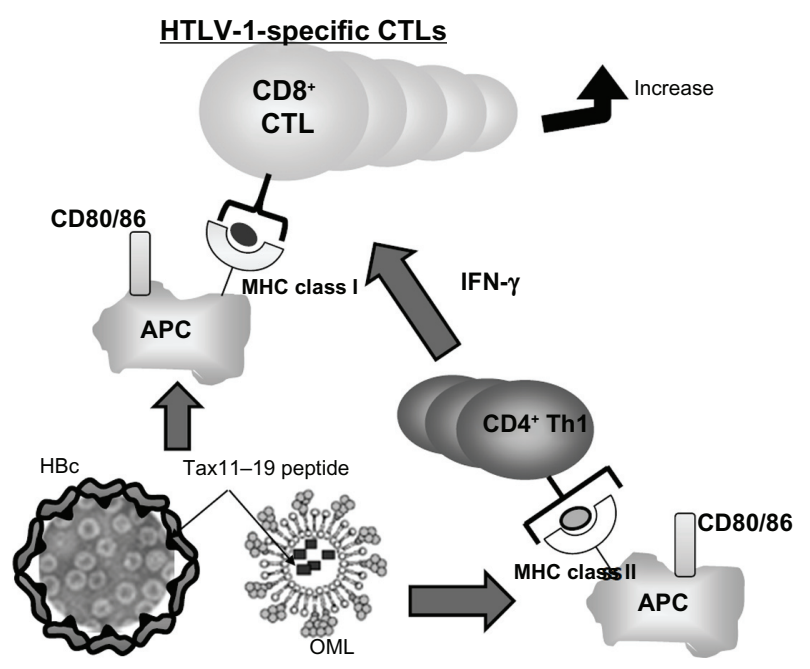

\section{HTLV-1 Tax/HBc chimeric vaccine OML/Tax vaccine}

Figure 4 Strategies targeted at inducing CTLs for the prophylaxis of infectious disease and therapeutic approaches for cancer.

Notes: HTLV-I Tax/HBc chimeric particles and OML/Tax can be phagocytosed by APCs and antigenic fragments presented by either MHC class I or II molecules to activate either $\mathrm{CD}^{+} \mathrm{T}$ cells or $\mathrm{CD} 4^{+} \mathrm{Th}$ l cells, respectively. Thus, this strategy could induce strong cellular immune responses without adjuvant as assessed by the increase of CD86 and MHC class I/II expression.

Abbreviations: APC, antigen-presenting cell; CTLs, cytotoxic T lymphocytes; HBC, hepatitis B core; HTLV, human T-cell leukemia virus- I; MHC, major histocompatibility complex; OMLs, oligomannose-coated liposomes; Th, T cell helper.

\section{Conclusion}

The recent emergence of targeting antigens specific to DCs and their subsequent activation with biomaterials has demonstrated an exciting potential for developing new vaccines. Nanoparticles such as liposomes and VLPs offer the ability to protect antigens from degradation and deliver them specifically to APCs. OML/Tax and HTLV-1 Tax/HBc chimeric particles are capable of inducing strong cellular immune responses without adjuvants and may be useful as effective carriers for prophylactic or therapeutic uses in infectious diseases and tumors such as HTLV-1-infected cells and ATL cells. Thus, novel chemical strategies can be employed to release antigens intracellularly where they can be processed by both MHC class I and class II pathways (Figure 4). Nanoparticles can also be used as synthetic adjuvants that use different mechanisms to induce DC maturation and initiate adaptive immune responses. Moreover, bioconjugation strategies can be used to attach molecular "danger signals" to particles to amplify the immune response while providing a potential nontoxic alternative adjuvant technology. Finally, the use of these very small nanoparticles offers an antigen-delivery system to unlock the potential of targeting lymph-node-resident DCs to induce adaptive immunity and therapeutic tolerance. The development of biomaterials for immune cell-targeting continues at a rapid pace, and future studies should focus on optimizing antigen delivery and adjuvant activity. These new approaches, inducing antitumor immunity and anti-allergic effects using vaccines, may provide a new prophylactic strategy for infectious disease and therapeutic approaches for cancer.

\section{Acknowledgments}

I would like to express my thanks to Dr Sato and Dr Shimizu from BioMedCore Inc (Yokohama, Japan), Dr Kino and Dr Fukada from The Chemo-Sero-Therapeutic Research Institute (Kumamoto, Japan), Dr Suzuki and Mr Toji from the Medical and Biological Laboratories Co, Ltd (Nagoya, Japan), and Dr Nishimura and Dr Hirata from Kumamoto University (Kumamoto, Japan). This work was supported in part by a Grant-in-Aid for Scientific Research from the Japan Society for the Promotion of Science and the Japan Leukemia Research Fund.

\section{Disclosure}

The authors declare no potential conflicts of interest relevant to this article. 


\section{References}

1. Muthu MS, Singh S. Targeted nanomedicines: effective treatment modalities for cancer, AIDS and brain disorders. Nanomedicine (Lond). 2009;4(1):105-118.

2. Albert ML, Sauter B, Bhardwaj N. Dendritic cells acquire antigen from apoptotic cells and induce class I-restricted CTLs. Nature. 1998;392(6671):86-89.

3. Kawakami Y, Fujita T, Kudo C, et al. Dendritic cell based personalized immunotherapy based on cancer antigen research. Front Biosci. 2008;13:1952-1958.

4. Kozako T. New strategy of adult T-cell leukemia treatment targeted for anti-tumor immunity and a longevity gene-encoded protein. Yakugaku Zasshi. 2011;131(7):1061-1072.

5. Yasunaga J, Sakai T, Nosaka K, et al. Impaired production of naive $\mathrm{T}$ lymphocytes in human T-cell leukemia virus type I-infected individuals: its implications in the immunodeficient state. Blood. 2001;97(10):3177-3183.

6. Harashima N, Kurihara K, Utsunomiya A, et al. Graft-versus-Tax response in adult T-cell leukemia patients after hematopoietic stem cell transplantation. Cancer Res. 2004;64(1):391-399.

7. Ikehara Y, Shiuchi N, Kabata-Ikehara S, et al. Effective induction of anti-tumor immune responses with oligomannose-coated liposome targeting to intraperitoneal phagocytic cells. Cancer Lett. 2008; 260(1-2):137-145.

8. Matsui M, Shimizu Y, Kodera Y, Kondo E, Ikehara Y, Nakanishi H. Targeted delivery of oligomannose-coated liposome to the omental micrometastasis by peritoneal macrophages from patients with gastric cancer. Cancer Sci. 2010;101(7):1670-1677.

9. Takagi H, Furuya N, Kojima N. Preferential production of IL-12 by peritoneal macrophages activated by liposomes prepared from neoglycolipids containing oligomannose residues. Cytokine. 2007;40(3):241-250.

10. Kozako T, Hirata S, Shimizu Y, et al. Oligomannose-coated liposomes efficiently induce human T-cell leukemia virus-1-specific cytotoxic T lymphocytes without adjuvant. FEBS J. 2011;278(8):1358-1366.

11. Grgacic EV, Anderson DA. Virus-like particles: passport to immune recognition. Methods. 2006;40(1):60-65.

12. Zhang S, Cubas R, Li M, Chen C, Yao Q. Virus-like particle vaccine activates conventional $\mathrm{B} 2$ cells and promotes $\mathrm{B}$ cell differentiation to IgG2a producing plasma cells. Mol Immunol. 2009;46(10):1988-2001.

13. Cui Z, Huang L. Liposome-polycation-DNA (LPD) particle as a carrier and adjuvant for protein-based vaccines: therapeutic effect against cervical cancer. Cancer Immunol Immunother. 2005;54(12): 1180-1190.

14. Whitmore MM, Li S, Falo L Jr, Huang L. Systemic administration of LPD prepared with CpG oligonucleotides inhibits the growth of established pulmonary metastases by stimulating innate and acquired antitumor immune responses. Cancer Immunol Immunother. 2001; 50(10):503-514.

15. Malyala P, O’Hagan DT, Singh M. Enhancing the therapeutic efficacy of $\mathrm{CpG}$ oligonucleotides using biodegradable microparticles. Adv Drug Deliv Rev. 2009;61(3):218-225.

16. Badiee A, Jaafari MR, Samiei A, Soroush D, Khamesipour A. Coencapsulation of $\mathrm{CpG}$ oligodeoxynucleotides with recombinant Leishmania major stress-inducible protein 1 in liposome enhances immune response and protection against leishmaniasis in immunized BALB/c mice. Clin Vaccine Immunol. 2008;15(4):668-674.

17. Li S, Huang L. In vivo gene transfer via intravenous administration of cationic lipid-protamine-DNA (LPD) complexes. Gene Ther. 1997;4(9): 891-900.

18. Yan W, Chen W, Huang L. Mechanism of adjuvant activity of cationic liposome: phosphorylation of a MAP kinase, ERK and induction of chemokines. Mol Immunol. 2007;44(15):3672-3681.

19. Chen W, Yan W, Huang L. A simple but effective cancer vaccine consisting of an antigen and a cationic lipid. Cancer Immunol Immunother. 2008;57(4):517-530.
20. Bhowmick S, Mazumdar T, Sinha R, Ali N. Comparison of liposome based antigen delivery systems for protection against Leishmania donovani. J Control Release. 2010;141(2):199-207.

21. Ikehara Y, Niwa T, Biao L, et al. A carbohydrate recognition-based drug delivery and controlled release system using intraperitoneal macrophages as a cellular vehicle. Cancer Res. 2006;66(17):8740-8748.

22. Kawamura K, Kadowaki N, Suzuki R, et al. Dendritic cells that endocytosed antigen-containing IgG-liposomes elicit effective antitumor immunity. J Immunother. 2006;29(2):165-174.

23. Hirai M, Minematsu H, Kondo N, Oie K, Igarashi K, Yamazaki N. Accumulation of liposome with Sialyl Lewis $\mathrm{X}$ to inflammation and tumor region: application to in vivo bio-imaging. Biochem Biophys Res Commun. 2007;353(3):553-558.

24. McGreal EP, Martinez-Pomares L, Gordon S. Divergent roles for C-type lectins expressed by cells of the innate immune system. Mol Immunol. 2004;41(11):1109-1121.

25. Robinson MJ, Sancho D, Slack EC, LeibundGut-Landmann S, Reis e Sousa C. Myeloid C-type lectins in innate immunity. Nat Immunol. 2006;7(12):1258-1265.

26. Adams EW, Ratner DM, Seeberger PH, Hacohen N. Carbohydratemediated targeting of antigen to dendritic cells leads to enhanced presentation of antigen to T cells. Chembiochem. 2008;9(2):294-303.

27. Turner MW. Mannose-binding lectin: the pluripotent molecule of the innate immune system. Immunol Today. 1996;17(11):532-540.

28. Geijtenbeek TB, Torensma R, van Vliet SJ, et al. Identification of DC-SIGN, a novel dendritic cell-specific ICAM-3 receptor that supports primary immune responses. Cell. 2000;100(5):575-585.

29. Stambach NS, Taylor ME. Characterization of carbohydrate recognition by langerin, a C-type lectin of Langerhans cells. Glycobiology. 2003; 13(5):401-410.

30. Singh SK, Stephani J, Schaefer M, et al. Targeting glycan modified OVA to murine DC-SIGN transgenic dendritic cells enhances MHC class I and II presentation. Mol Immunol. 2009;47(2-3):164-174.

31. Ishii M, Koyama A, Iseki H, Narumi H, Yokoyama N, Kojima N. Anti-allergic potential of oligomannose-coated liposome-entrapped Cry j 1 as immunotherapy for Japanese cedar pollinosis in mice. Int Immunopharmacol. 2010;10(9):1041-1046.

32. Kojima N, Biao L, Nakayama T, Ishii M, Ikehara Y, Tsujimura K. Oligomannose-coated liposomes as a therapeutic antigen-delivery and an adjuvant vehicle for induction of in vivo tumor immunity. J Control Release. 2008;129(1):26-32.

33. Nishikawa Y, Zhang H, Ikehara Y, Kojima N, Xuan X, Yokoyama N. Immunization with oligomannose-coated liposome-entrapped dense granule protein 7 protects dams and offspring from Neospora caninum infection in mice. Clin Vaccine Immunol. 2009;16(6):792-797.

34. Shimizu Y, Takagi H, Nakayama T, et al. Intraperitoneal immunization with oligomannose-coated liposome-entrapped soluble leishmanial antigen induces antigen-specific T-helper type immune response in $\mathrm{BALB} / \mathrm{c}$ mice through uptake by peritoneal macrophages. Parasite Immunol. 2007;29(5):229-239.

35. Janeway CA Jr, Medzhitov R. Innate immune recognition. Annu Rev Immunol. 2002;20:197-216.

36. Kawai T, Akira S. Pathogen recognition with Toll-like receptors. Curr Opin Immunol. 2005;17(4):338-344.

37. Kato C, Kajiwara T, Numazaki M, Takagi H, Kojima N. Oligomannosecoated liposomes activate ERK via Src kinases and PI3K/Akt in J774 A.1 cells. Biochem Biophys Res Commun. 2008;372(4): 898-901.

38. Kojima N, Kawauchi Y, Ishii M. Development of novel carbohydratecoated liposome-based vaccines. Trends Glycosci Glycotechnol. 2011;23(134):257-271.

39. Mizuuchi M, Hirohashi Y, Torigoe T, et al. Novel oligomannose liposome-DNA complex DNA vaccination efficiently evokes anti-HPV E6 and E7 CTL responses. Exp Mol Pathol. 2012;92(1):185-190.

40. Ishii M, Kojima N. Mucosal adjuvant activity of oligomannose-coated liposomes for nasal immunization. Glycoconj J. 2010;27(1):115-123. 
41. Hinuma Y, Nagata K, Hanaoka M, et al. Adult T-cell leukemia: antigen in an ATL cell line and detection of antibodies to the antigen in human sera. Proc Natl Acad Sci U S A. 1981;78(10):6476-6480.

42. Poiesz BJ, Ruscetti FW, Gazdar AF, Bunn PA, Minna JD, Gallo RC. Detection and isolation of type $\mathrm{C}$ retrovirus particles from fresh and cultured lymphocytes of a patient with cutaneous T-cell lymphoma. Proc Natl Acad Sci US A. 1980;77(12):7415-7419.

43. Tsukasaki K, Hermine O, Bazarbachi A, et al. Definition, prognostic factors, treatment, and response criteria of adult T-cell leukemialymphoma: a proposal from an international consensus meeting. J Clin Oncol. 2009;27(3):453-459.

44. Taylor GP, Matsuoka M. Natural history of adult T-cell leukemia/ lymphoma and approaches to therapy. Oncogene. 2005;24(39): 6047-6057.

45. Tsukasaki K, Utsunomiya A, Fukuda H, et al. VCAP-AMP-VECP compared with biweekly CHOP for adult T-cell leukemia-lymphoma: Japan Clinical Oncology Group Study JCOG9801. J Clin Oncol. 2007;25(34):5458-5464.

46. Yamada Y, Tomonaga M, Fukuda H, et al. A new G-CSF-supported combination chemotherapy, LSG15, for adult T-cell leukaemialymphoma: Japan Clinical Oncology Group Study 9303. Br J Haematol. 2001;113(2):375-382.

47. Yoshida M. Molecular approach to human leukemia: isolation and characterization of the first human retrovirus HTLV-1 and its impact on tumorigenesis in adult T-cell leukemia. Proc Jpn Acad Ser B Phys Biol Sci. 2010;86(2):117-130.

48. Yasunaga J, Matsuoka M. Leukaemogenic mechanism of human T-cell leukaemia virus type I. Rev Med Virol. 2007;17(5):301-311.

49. Jacobson S, Shida H, McFarlin DE, Fauci AS, Koenig S. Circulating $\mathrm{CD}^{+}$cytotoxic T lymphocytes specific for HTLV-I pX in patients with HTLV-I associated neurological disease. Nature. 1990;348(6298): 245-248

50. Bangham CR. HTLV-1 infection: role of CTL efficiency. Blood. 2008;112(6):2176-2177.

51. Kozako T, Akimoto M, Toji S, et al. Target epitopes of HTLV-1 recognized by class I MHC-restricted cytotoxic T lymphocytes in patients with myelopathy and spastic paraparesis and infected patients with autoimmune disorders. J Med Virol. 2011;83(3):501-509.
52. Kozako T, Arima N, Toji S, et al. Reduced frequency, diversity, and function of human $\mathrm{T}$ cell leukemia virus type 1 -specific $\mathrm{CD}^{+} \mathrm{T}$ cell in adult T cell leukemia patients. J Immunol. 2006;177(8):5718-5726.

53. Kozako T, Yoshimitsu M, Akimoto M, et al. Programmed death-1 (PD-1)/PD-1 ligand pathway-mediated immune responses against human T-lymphotropic virus type 1 (HTLV-1) in HTLV-1-associated myelopathy/tropical spastic paraparesis and carriers with autoimmune disorders. Hum Immunol. 2011;72(11):1001-1006.

54. Kozako T, Yoshimitsu M, Fujiwara H, et al. PD-1/PD-L1 expression in human T-cell leukemia virus type 1 carriers and adult T-cell leukemia/ lymphoma patients. Leukemia. 2009;23(2):375-382.

55. Ohashi T, Hanabuchi S, Kato H, et al. Prevention of adult T-cell leukemialike lymphoproliferative disease in rats by adoptively transferred $\mathrm{T}$ cells from a donor immunized with human T-cell leukemia virus type 1 Tax-coding DNA vaccine. J Virol. 2000;74(20):9610-9616.

56. Fukasawa M, Shimizu Y, Shikata K, et al. Liposome oligomannosecoated with neoglycolipid, a new candidate for a safe adjuvant for induction of $\mathrm{CD}^{+}$cytotoxic T lymphocytes. FEBS Lett. 1998;441(3): 353-356.

57. Sugimoto M, Ohishi K, Fukasawa M, et al. Oligomannose-coated liposomes as an adjuvant for the induction of cell-mediated immunity. FEBS Lett. 1995;363(1-2):53-56.

58. Milich DR, McLachlan A, Thornton GB, Hughes JL. Antibody production to the nucleocapsid and envelope of the hepatitis B virus primed by a single synthetic T cell site. Nature. 1987;329(6139):547-549.

59. Storni T, Lechner F, Erdmann I, et al. Critical role for activation of antigen-presenting cells in priming of cytotoxic $\mathrm{T}$ cell responses after vaccination with virus-like particles. J Immunol. 2002;168(6): 2880-2886.

60. Kozako T, Fukada K, Hirata S, et al. Efficient induction of human T-cell leukemia virus-1-specific CTL by chimeric particle without adjuvant as a prophylactic for adult T-cell leukemia. Mol Immunol. 2009;47(2-3): 606-613.
International Journal of Nanomedicine

\section{Publish your work in this journal}

The International Journal of Nanomedicine is an international, peerreviewed journal focusing on the application of nanotechnology in diagnostics, therapeutics, and drug delivery systems throughout the biomedical field. This journal is indexed on PubMed Central,

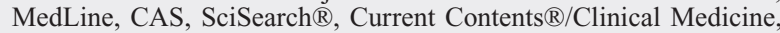

\section{Dovepress}

Journal Citation Reports/Science Edition, EMBase, Scopus and the Elsevier Bibliographic databases. The manuscript management system is completely online and includes a very quick and fair peer-review system, which is all easy to use. Visit http://www.dovepress.com/ testimonials.php to read real quotes from published authors. 